\title{
EFECTO DE LA SUPLEMENTACIÓN CON ENSILAJE DE NARANJA SOBRE LA COMPOSICIÓN DE LA LECHE BOVINA
}

\author{
EFFECT OF ORANGE SILAGE SUPPLEMENTATION \\ ON BOVINE MILK COMPOSITION
}

\section{${ }^{1}$ Dixon Fabián Flórez Delgado ${ }^{2}$ Alfonso Eugenio Capacho Mogollón ${ }^{3}$ Sandra Milena Quintero Muiño, ${ }^{4}$ Pavlova Báez Gamboa}

\author{
${ }^{1}$ Magister en Sistemas Sostenibles de Producción, Universidad de Pamplona. \\ 2Magister en Calidad de la Educación Superior, Universidad de Pamplona. \\ ${ }^{3}$ Especialista en Nutrición Animal Sostenible, Universidad de Pamplona. \\ ${ }^{4}$ Zootecnista, Universidad de Pamplona. \\ ${ }^{1}$ dixon.florez@unipamplona.edu.co; ${ }^{2}$ aecapacho@unipamplona.edu.co; \\ ${ }^{3}$ sandra.quintero@unipamplona.edu.co
}

\section{RESUMEN}

Contextualización: en la producción ganadera, la suplementación alimenticia se lleva a cabo mediante alimentos balanceados comerciales que incrementan los costos de producción y afectan los márgenes de rentabilidad de las empresas pecuarias.

Vacío de conocimiento: el ensilaje de naranja se ve como una alternativa nutricional y económica para las empresas ganaderas que están en busca de su sostenibilidad bajo los siguientes interrogantes: ¿cuál es el efecto del ensilaje de naranja sobre la productividad y calidad de leche bovina?, ¿es posible reducir los costos por concepto de alimentación al incluir este ensilaje y reducir el alimento balanceado sin afectar los parámetros productivos en bovinos leche?
Propósito del estudio: evaluar el efecto del suministro de ensilaje de naranja (EN) sobre la composición de la leche bovina en la Granja Experimental Villa Marina de la Universidad de Pamplona.

Metodología: se utilizó un diseño de bloques completos aleatorizados con 12 animales cuya genética está influenciada por las razas Gyr, Holstein, Jersey y Rojo Sueco, distribuidos en un tratamiento con la sustitución del $20 \%$ del alimento balanceado comercial (T1) y un testigo (T2). Se elaboró el EN con frutos producidos en la granja con 30 días de fermentación. Se realizó un periodo de acostumbramiento de 10 días. Se midió la composición de la leche (grasa, proteína, sólidos no grasos, minerales y lactosa) cada siete días durante 90 días. 
Los datos obtenidos se sometieron a un análisis de varianza y de correlación.

Resultados y conclusiones: la inclusión de EN generó un mayor contenido de grasa $(4,36 \%$, $\mathrm{P}<0,05)$ en la leche de las vacas en comparación con el tratamiento testigo (3,75\%). No hubo efecto de los tratamientos en los demás componentes de la leche evaluados. La producción diaria de leche no fue afectada $(P>0,05)$ por los tratamientos. El costo de suplementación por litro de leche se estimó en COP 265,15. La sustitución de alimento balanceado comercial en un $20 \%$ con EN mejora el porcentaje de grasa en la leche y disminuye los costos de la alimentación respecto al manejo convencional, constituyéndose en una alternativa sostenible de alimentación en vacas lactantes cruzadas.

Palabras clave: cítrico; fermentación anaeróbica; grasa de la leche; rumiante.

\section{ABSTRACT}

Contextualization: In livestock production, food supplementation is carried out with commercial feed that increases production costs and thus affects the profit margins of livestock companies.

Knowledge gap: Orange silage is considered a nutritional and economic alternative for livestock companies in search of sustainability under the following questions: What is the effect of orange silage on the productivity and quality of bovine milk? Is it possible to reduce the feeding costs by including this silage and reducing the feed without affecting the productive parameters in milk cattle?
Purpose: The main objective of this study was to evaluate the effect of orange silage (EN) supply on bovine milk composition at Villa Marina Experimental Farm of University of Pamplona.

Methodology: A randomized complete block design was used with 12 animals whose genetics are influenced by the Gyr, Holstein, Jersey and Swedish Red breed, distributed in a treatment with the substitution of $20 \%$ of commercial balanced food (T1) and a control (T2). The EN was elaborated with fruits produced on the farm with 30 days of fermentation. A period of 10 days of accustoming was carried out. The milk composition (fat, protein, non-fat solids (SNG), minerals and lactose) were measured every seven days for 90 days. The data obtained were subjected to an analysis of variance and correlation.

Results and conclusions: The inclusion of EN produced a higher fat content (4,36\%, $\mathrm{P}<0.05)$ in the cow's milk, compared to the control treatment (3.75\%). There was no effect of treatments on the remainder milk components evaluated. The daily production of milk was not affected $(P>0.05)$ by treatments. The replacement of commercial balanced food by $20 \%$ with EN improves the milk fat content and decreases the costs of feeding, compared to conventional management, becoming a sustainable feeding alternative in crossbreed lactating cows.

Keywords: citrus; anaerobic fermentation; milk fat; ruminant. 


\section{RESUMEN GRÁFICO}

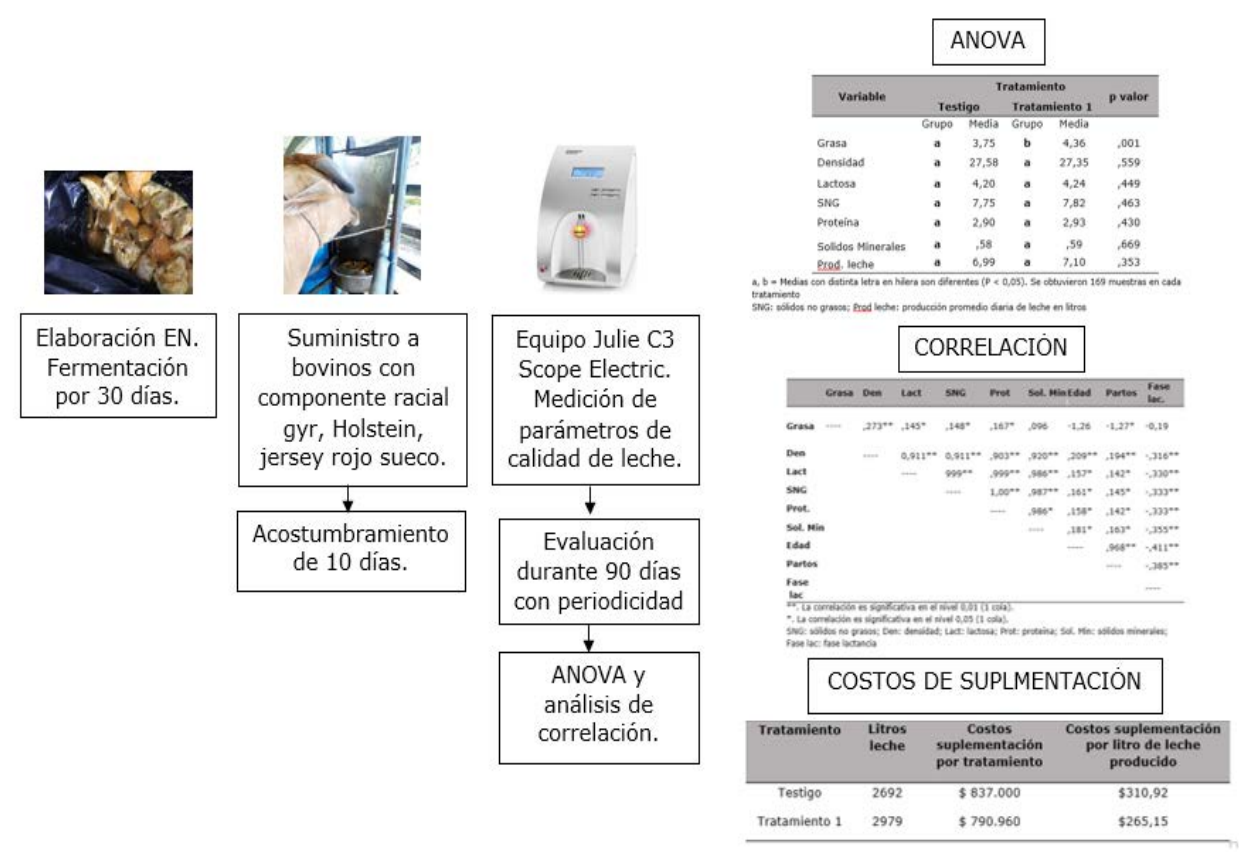

\section{INTRODUCCIÓN}

Los forrajes son la base de la alimentación en los sistemas de producción de rumiantes, y en algunos casos es necesario la utilización de los alimentos balanceados para llenar el requerimiento nutricional de los animales, lo cual reduce los márgenes de rentabilidad por el incremento en los costos de alimentación (Bermúdez, et al., 2015).

Los alimentos balanceados, que están formulados a base de granos y cereales importados, se convierten en el principal suplemento alimenticio disponible para los productores en el país y generan incrementos notables en los costos de producción. Por esto, muchos productores han incursionado en la formulación y elaboración de su propio suplemento alimenticio, utilizando nuevas alternativas de alimentación a bajo costo (Triana, et al., 2014), a partir de subproductos agroindustriales y residuos de cosecha, como fuentes de alimento, especialmente en regiones donde la oferta de forraje en pastoreo es restrictiva por diversas factores (i.e. época de sequía) (Martínez, et al., 2008), constituyéndose en opciones para generar una sostenibilidad económica en los sistemas de producción (Pereira, et al., 2008).

La naranja (fruto entero) es un recurso local disponible en la provincia de Pamplona (Norte de Santander) cuyo uso, debido a su bajo valor comercial, se ha direccionado a la alimentación de rumiantes. Posee nutrientes y una alta digestibilidad de la materia seca (58\%), lo que lo convierte en una alternativa viable para la alimentación de rumiantes (Velásquez, Esquivel, Montero y Ku, 2012).

La naranja para alimentación animal puede emplearse deshidratada, peletizada o molida; sin embargo, la literatura sugiere que la mejor forma de conservarla y usarla es en forma húmeda a través del ensilaje (Ítalvo, et al, 2000). Los carbohidratos solubles (azúcares simples) y estructurales (celulosa, hemicelulosa y pectina) son sus principales aportes nutricionales, de fácil fermentación en el rumen que favorecen la formación de ácidos propiónico y acético respectivamente (Fernández, 2014). El uso de este fruto en la alimentación animal, además de reducir los 
problemas de contaminación por la producción de malos olores, proliferación de insectos e incluso filtraciones ácidas en el suelo, ha demostrado reducir la presencia de E. coli y Salmonella en el intestino de los rumiantes, mejorando el estado de salud de estos (Domínguez, 2013) al proveer fibra, vitaminas y aceites esenciales que actúan como un antibiótico natural.

La alimentación de rumiantes con frutos de naranja y subproductos cítricos disminuye la dependencia y uso de cereales en la alimentación animal con el uso aquellos frutos que no cumplen con el estándar mínimo para su comercialización (Pássaro, et al., 2012) y el efecto negativo de los residuos agroindustriales al ambiente (Grasser, 1995; Ajila, 2012). El objetivo del presente estudio fue evaluar el efecto de la suplementación con ensilaje de naranja sobre los parámetros de calidad de la leche bovina.

\section{MATERIALES Y MÉTODOS Ubicación}

El estudio se realizó en la Granja Experimental Villa Marina de la Universidad de Pamplona, ubicada en la vereda Matajira, municipio de Pamplonita. Tiene una altitud de 1100 m.s.n.m. en su parte baja y de 1800 en la parte alta; su temperatura promedio es de $20^{\circ} \mathrm{C}$, topografía pendiente, con una precipitación de $1400 \mathrm{~mm}$ anual. El experimento, se realizó en los meses de julio y agosto de 2018.

\section{Animales y manejo de la alimentación}

Se emplearon doce vacas lactantes cuya composición racial estuvo conformada por Holstein, Gyr, Jersey y Rojo Sueco, con edades entre los 3 y los 11 años, y un peso promedio de $425 \mathrm{Kg}$. Se dividieron de manera aleatoria en dos grupos, siendo identificadas con un collar que permitió hacer el seguimiento semanal para la toma de datos y diligenciamiento de registros.
El manejo alimenticio consistió en pastoreo rotacional, con forraje estrella (Cynodon plectostachyus) y braquiaria común (Brachiaria decumbens) con periodos de ocupación de 2 días y de descanso de 42 días, con suplemento de 60 gramos de sal y un kilogramo de alimento balanceado comercial por animal / día.

\section{Elaboración del ensilaje de naranja}

Se utilizó la naranja producida en la Granja Experimental Villa Marina, posteriormente se picó en trozos pequeños (indicar tamaño y con qué equipo o herramienta realizó el picado). Se almacenó el material en silo de bolsa (Fernández, 2015) con un calibre 7 con capacidad para $50 \mathrm{~kg}$. Para el proceso de elaboración se alternaron capas de naranja picada de un espesor de $10 \mathrm{~cm}$ seguida de una capa de harina de arroz para reducir los niveles de humedad hasta completar el total de la bolsa (Triana, et al. 2014). Se empleó un $97 \%$ de naranja y un $3 \%$ de harina de arroz. Las bolsas se almacenaron en un lugar protegido del sol y de los roedores para su proceso de fermentación de 21 días (Bermúdez et al., 2015).

\section{Tratamientos}

Se evaluaron dos tratamientos: el tratamiento experimental, que consistió en sustituir el $20 \%$ del alimento balanceado comercial por ensilaje de naranja y el tratamiento testigo, que consistió en alimento balanceado convencional. Se implementó un periodo de acostumbramiento de los bovinos al ensilaje de naranja durante diez días (Velásquez, et al., 2012) para iniciar con la toma de datos.

\section{Mediciones}

Calidad nutricional de los alimentos. Se determinó su composición nutricional de este suplemento alimenticio, teniendo en cuenta lo siguientes parámetros nutricionales: 
- $\mathrm{pH}$, a través de potenciometría

- Materia Seca, por medio de termo gravimétrico a $103^{\circ} \mathrm{C} \pm 2^{\circ} \mathrm{C}$

- Proteína, por Kjeldahl

- Cenizas por incineración directa a $600^{\circ} \mathrm{C}$

- Grasa, por extracción

- Fibra Cruda, por método gravimétrico

Producción y calidad de leche: pasado el periodo de acostumbramiento de diez días al EN, se inició con la medición de la calidad de la leche empleando el equipo Julie C3 Scope Electric con intervalos de siete días durante todo el periodo experimental (60 días). Se tomó una muestra de leche en el ordeño de la mañana (7:00 am) para medición de sólidos totales.

\section{Diseño experimental y análisis estadístico} Se aplicaron pruebas de estadística descriptiva, análisis de varianza mediante la prueba de Tukey $(P<0,05)$ y análisis de correlación de Pearson para determinar el efecto y las diferencias entre el tratamiento y el testigo como suplemento alimenticio sobre los parámetros de calidad de leche bovina. Se siguió el siguiente diseño experimental (ecuación 1):

$Y i j=\mu+T i+E j+$ Covariable (ecuación 1)
Donde:

Y: producción y calidad de la leche

$\mu$ : media general

Ti: efecto del tratamiento

Ej: error general

Covariable: raza, peso, edad, lactancia

\section{RESULTADOS Y DISCUSIÓN}

La composición nutricional del ensilaje de naranja se indica en la tabla 1.

Tabla 1. Composición nutricional del ensilaje de naranja.

\begin{tabular}{l|c} 
Composición & \% \\
\hline $\mathrm{pH}$ & 3,63 \\
\hline Materia Seca & 13,04 \\
\hline Proteína & 8,42 \\
\hline Cenizas & 0,56 \\
\hline Grasa & 1,59 \\
\hline Fibra Cruda & 32,88 \\
\hline
\end{tabular}

Fuente: Autores

\section{Producción y composición de la leche}

En cuanto a la producción diaria de leche, no se presentaron diferencias significativas $(P>0,05)$, con medias de 6,99 litros por animal para el testigo y 7,10 para el tratamiento 1 (Tabla 2). 
Tabla 2. Producción y composición de la leche de vacas cruzadas con la sustitución del $20 \%$ del alimento balanceado comercial por ensilaje de naranja (T1) y alimento balanceado de manera convencional (testigo).

\begin{tabular}{|c|c|c|c|}
\hline \multirow{2}{*}{ Variable } & \multicolumn{2}{|c|}{ Tratamiento } & \multirow{2}{*}{ Valor $\mathbf{P}$} \\
\hline & Testigo & Tratamiento 1 & \\
\hline Producción de leche (I/vaca/día) & $6,99 \pm 0,12$ & $7,1 \pm 0,09$ & 0,35 \\
\hline \multicolumn{4}{|l|}{ Composición (\%) } \\
\hline Grasa & $3,75 \pm 0,06 a$ & $4,36 \pm 0,07 b$ & 0,001 \\
\hline Lactosa & $4,20 \pm 0,08 a$ & $4,24 \pm 0,10 a$ & 0,44 \\
\hline SNG & $7,75 \pm 0,21 a$ & $7,82 \pm 0,19 a$ & 0,46 \\
\hline Proteína & $2,90 \pm 0,04 a$ & $2,93 \pm 0,07 a$ & 0,43 \\
\hline Minerales & $0,58 \pm 0,01 a$ & $0,59 \pm 0,03 a$ & 0,66 \\
\hline Densidad (g/ml) & $1,027 \pm 0,02 a$ & $1,027 \pm 0,01 a$ & 0,55 \\
\hline
\end{tabular}

SNG: sólidos no grasos. Medias con distinta letra en la misma fila indican diferencias significativas $(P<0,05)$.

Fuente: Autores

El contenido de grasa en la leche fue mayor $(4,36 \%, \mathrm{P}<0,05)$ en las vacas alimentadas con el tratamiento 1 , en comparación con el testigo. Esto puede estar explicado por el aporte de fibra que realiza el ensilaje de avena, que produce acetato a nivel ruminal, el cual es precursor de ácidos grasos de hasta 16 carbonos (Domínguez, 2013).

La inclusión de EN en la dieta de rumiantes ha sido asociada a efectos positivos, especialmente en relación con la fermentación ruminal (Flórez, et al. 2018) y con los aumentos en el contenido graso de la leche (Wing, 1982; Belibasakis y Tsirgogianni, 1996), fermentación de pectinas (Strobel y Russell, 1986) y cambios moderados en el $\mathrm{pH}$ del rumen (Villareal, 2006; Piquer, 2009), evitando la presencia de acidosis (Wing, 1982). Todo lo anterior favorece el empleo de este suplemento como sustituto de los alimentos balanceados comerciales al ser una fuente de energía alternativa.

No se presentaron diferenciasen el contenido de proteína, lactosa, minerales y densidad de la leche por efecto de los tratamientos $(P>0,05)$. En la tabla 3 se muestra el nivel de correlación entre las variables asociadas a la calidad de la leche, donde se logra determinar que existe una correlación alta positiva entre los componentes lactosa y sólidos no grasos $(R=0,9)$, lactosa y proteína $(R=0,9)$, lo que indica que, a medida que la lactosa aumentó, el contenido de sólidos no grasos y proteína fue mayor; asimismo, existe una relación lineal fuerte entre sólidos no grasos y proteína $(1,0)$, ya que, a medida que el contenido de sólidos no grasos aumentó, la proteína se comportó de la misma manera. 
Tabla 3. Coeficientes de correlación de Pearson entre las variables de calidad de la leche, edad, número de partos y fase de la lactancia, número de partos y fase de la lactancia

\begin{tabular}{l|r|r|r|r|r|r|r|r|r|}
\hline & \multicolumn{2}{|c|}{ Grasa } & Den. & Lact. & SNG & Prot. & Sol. Min & Edad & Partos \\
Lac.
\end{tabular}

**. La correlación es significativa en el nivel 0,01 (1 cola). *. La correlación es significativa en el nivel 0,05 (1 cola). SNG: sólidos no grasos; Den: densidad; Lact: lactosa; Prot: proteína; Sol. Min: sólidos minerales; Fase lac.: fase lactancia. Fuente: Autores

También se puede observar en la correlación de Pearson que no existe una relación entre las variables densidad, lactosa y sólidos minerales $(-, 145$ y 0,096 respectivamente), ya que el valor de $r$ es relativamente cercano a 0 , lo que indica que el contenido de lactosa y sólidos minerales no influencian en mayor medida la densidad.

Finalmente, los factores de edad, número de partos y fase de la lactancia presentaron una correlación negativa, ya que, al aumentar estos factores, los parámetros de calidad de la leche, como la grasa, disminuyen.

Los costos de suplementación con EN por litro de leche producido se calcularon en COP 253,59 y COP 300 para el tratamiento 1 y testigo respectivamente como se muestra en la siguiente tabla 4.

Tabla 4. Costo de suplementación de litro de leche producido con ensilaje de naranja.

\begin{tabular}{|l|c|c|c|}
\hline & Litros leche & $\begin{array}{c}\text { Costos suplementación } \\
\text { por tratamiento }\end{array}$ & $\begin{array}{c}\text { Costos suplementación por } \\
\text { itro de leche producido }\end{array}$ \\
\hline Testigo & 2692 & COP 837.000 & COP 310,92 \\
\hline Tratamiento 1 & 2979 & COP 790.960 & COP 265,15 \\
\hline
\end{tabular}

Fuente: Autores

\section{CONCLUSIONES}

La sustitución de alimento balanceado comercial en un $20 \%$ con EN, mejora el porcentaje de grasa en la leche y disminuye los costos de la alimentación (indicar el porcentaje) respecto al manejo convencional, constituyéndose en una alternativa sostenible de alimentación en vacas lactantes cruzadas. 


\section{CONTRIBUCIÓN DE LA AUTORÍA}

Primer autor. Metodología, análisis de datos, escritura borrador original. Segundo autor. Investigación, conceptualización, escritura borrador original. Tercer autor. Metodología, investigación, escritura borrador original. Cuarto autor. Investigación, escritura borrador original.

\section{AGRADECIMIENTOS}

Los autores expresan su agradecimiento a la Universidad de Pamplona, en especial a la Vicerrectoría de Investigaciones por su colaboración en el desarrollo del proyecto a través del Semillero de Investigación en Producción Animal Sostenible SIPAS del programa de Zootecnia. De la misma manera, al proyecto "Efecto de la suplementación con ensilaje de naranja sobre la calidad de leche caprina", desarrollado por investigadores de la Universidad de Pamplona en la Granja Experimental Villa Marina en el año 2017, cuyos resultados fueron claves para el desarrollo de la presente investigación.

\section{LITERATURA CITADA}

Ajila, C. (2012). Sustainable solutions for agro processing waste management: An overview. In: Malik, A.; Grohmann, E. (Ed). Environmental Protection Strategies for Sustainable Development. Netherlands: Springer, 2012. p. 65109.

Belibasakis, N., y Tsirgogianni, D. (1996). Effects of dried citrus pulp on milk yield, milk composition and blood components of dairy cows. Animal Feed Science and Technology, 60(1), 87-92. https://doi.org/10.1016/0377-8401(95)00927-2

Bermúdez, L., Melo, E. y Estrada, J. (2015). Evaluación de ensilaje de naranja entera (Citrus sinensis) como alternativa de suplementación en bovinos. Revista Veterinaria y Zootecnia, 9(2), 38-53.

Domínguez, M. (2013). Efecto de la incorporación de pulpa de naranja en la dieta de cabras lecheras sobre la producción, composición de la leche y rendimiento quesero. Universidad Politécnica de Valencia, España. Tesis de Maestría.

Fernández, A. (2015). Subproductos de los Cítricos. Sitio argentino de Producción Animal. Argentina. p. 3.
Fernández, A. (2014). Transformación de subproductos y residuos de agroindustria de cultivos templados, subtropicales y tropicales en carne y leche bovina. Instituto Nacional de Tecnología Agropecuaria. Centro Regional Buenos Aires Sur Estación Experimental Agropecuaria Bordenave EEA Bordenave. $1^{\circ}$ edición. pp. 200.

Flórez, D., Capacho, A., Quintero, M. \& Gamboa, K. (2018). Efecto de la suplementación con ensilaje de naranja sobre la calidad de leche caprina. $R e-$ vista U.D.C.A Actualidad \& Divulgación Científica, 21(2), 501-506. https://doi.org/10.31910/rudca. v21.n2.2018.982

Grasser, L. (1995). Quantity and economic importance of nine selected by-products used in California dairy rations. Journal Dairy Science, 78(4), 962-971.

Martínez, J., Chongo, B., Jordán, H., Hernández, N., Fontes, D., Lezcano, Y., y Cubillas, N. (2008). Características nutritivas de los hollejos húmedos de naranja (Citrus sinensis cv. Valencia) mantenidos en estibas. Técnica Pecuaria en México, vol. 46, núm. 2. pp. 183-193. Instituto Nacional de Investigaciones Forestales, Agrícolas y Pecuarias Mérida, México.

Pássaro, C., Navarro, P., y Salvador, A. (2012). Poscosecha. In: Garcés, L.F. (Ed.). Cítricos: cultivo, cosecha e industrialización. Caldas: Corporación Universitaria Lasallista pp. 223-284.

Pereira, M., De azambuja, E., Yurika, I., da Rocha, M., Tiemi, J., y Yukio, E. (2008). Consumo de nutrientes e desempenho de cordeiros em confinamento alimentados com dietas com polpa cítrica úmida prensada em substituicão à silagem de milho. Revista Brasileira Zootecnia, 37, 134-139.

Piquer, O. (2009). Whole citrus fruits as an alternative to wheat grain or citrus pulp in sheep diet: Effect on the evolution of ruminal parameters. Small Ruminant Research, 83(1), 14-21.

Strobel, H., y Russell, J. (1986). Effect of pH and energy spilling on bacterial protein synthesis by carbohydrate-limited cultures of mixed rumen bacteria. Journal of Dairy Science, 69(11), 2941-2947.

Triana, E., Campo, Y., y Lizcano, H. (2014). Evaluación de ensilaje a partir de dos subproductos agroindustriales (cascara de naranja y plátano de rechazo) para alimentación de ganado bovino. Revista Alimentos Hoy, 22(31), 33-45. 
Velásquez, R., Esquivel, H., Montero, L., y Ku, J. (2012). Engorda de corderos Pelibuey con ensilaje de pulpa de naranja Citrus sinensis L. en jaulas elevadas. Revista Colombiana de Ciencia Animal, 5(1), 67-31.

Villareal, M. (2006). Effect of supplementation with pelleted citrus pulp on digestibility and intake in beef cattle fed a tropical grass-based diet (Cynodon nlemfuensis). Animal Feed Science and Technology, 125,163-173.

Wing, J. (1982). Citrus feed stuffs for dairy cattle. Gainesville: University of Florida. 
